\title{
The effect of iterative reconstruction on computed tomography assessment of emphysema, air trapping and airway dimensions
}

\author{
Onno M. Mets • Martin J. Willemink • \\ Freek P. L. de Kort • Christian P. Mol • Tim Leiner • \\ Matthijs Oudkerk • Mathias Prokop • Pim A. de Jong
}

Received: 13 February 2012 /Revised: 20 March 2012 / Accepted: 11 April 2012 /Published online: 23 May 2012

(C) The Author(s) 2012. This article is published with open access at Springerlink.com

\begin{abstract}
Objectives To determine the influence of iterative reconstruction (IR) on quantitative computed tomography (CT) measurements of emphysema, air trapping, and airway wall and lumen dimensions, compared to filtered back-projection (FBP).

Methods Inspiratory and expiratory chest CTs of 75 patients (37 male, 38 female; mean age $64.0 \pm 5.7$ years) were reconstructed using FBP and IR. CT emphysema, $\mathrm{CT}$ air trapping and airway dimensions of a segmental bronchus were quantified using several commonly used quantification methods. The two algorithms were compared using the concordance correlation coefficient $\left(p_{\mathrm{c}}\right)$ and Wilcoxon signed rank test.

Results Only the E/I-ratio ${ }_{\mathrm{MLD}}$ as a measure of CT air trapping and airway dimensions showed no significant differences between the algorithms, whereas all CT emphysema and the other $\mathrm{CT}$ air trapping measures were significantly different at IR when compared to FBP $(P<0.001)$.
\end{abstract}

O. M. Mets • M. J. Willemink $(\bowtie) \cdot$ F. P. L. de Kort • T. Leiner •

P. A. de Jong

Department of Radiology, UMC Utrecht,

P.O. Box 85500, E01.132, 3508 GA, Utrecht, the Netherlands

e-mail: m.willemink@umcutrecht.nl

C. P. Mol

Utrecht University Medical Center, Image Sciences Institute,

Utrecht, the Netherlands

M. Oudkerk

Department of Radiology, Groningen University Medical Center, Groningen, the Netherlands

M. Prokop

Department of Radiology, Nijmegen University Medical Center, Nijmegen, the Netherlands
Conclusion The evaluated IR algorithm significantly influences quantitative CT measures in the assessment of emphysema and air trapping. However, the E/I-ratio ${ }_{M L D}$ as a measure of CT air trapping, as well as the airway measurements, is unaffected by this reconstruction method. Quantitative $\mathrm{CT}$ of the lungs should be performed with careful attention to the CT protocol, especially when iterative reconstruction is introduced.

Key Points

- New techniques in CT allow numerous quantitative measurements of lung function.

- Iterative reconstruction influences quantitative CT measurements of emphysema and air trapping.

- Expiratory-to-inspiratory ratio of mean lung density and airway measurements are unaffected by iterative reconstruction.

- Quantitative lung-CT should be performed with careful attention to the CT protocol.

Keywords Computed tomography $\cdot$ Iterative reconstruction $\cdot$ Pulmonary emphysema $\cdot$ Chronic obstructive pulmonary disease $\cdot$ Quantitative CT

$\begin{array}{ll}\begin{array}{ll}\text { Abbreviations and acronyms } \\ \text { COPD }\end{array} & \begin{array}{l}\text { chronic obstructive pulmonary disease } \\ \text { computed tomography }\end{array} \\ \text { CT } & \begin{array}{l}\text { expiratory to inspiratory ratio of the } \\ \text { mean lung density }\end{array} \\ \text { E/I-ratio } & \text { percentage of voxels below }-856 \mathrm{HU} \\ \text { EXP }_{-856} & \begin{array}{l}\text { in expiratory CT } \\ \text { filtered back-projection }\end{array} \\ \text { FBP } & \text { Hounsfield unit }\end{array}$




\begin{tabular}{|c|c|}
\hline $\mathrm{IN}_{-950}$ & $\begin{array}{l}\text { percentage of voxels below }-950 \mathrm{HU} \\
\text { in inspiratory CT }\end{array}$ \\
\hline IR & iterative reconstruction \\
\hline LA & lumen area \\
\hline NELSON trial & $\begin{array}{l}\text { Nederlands Leuvens Longkanker } \\
\text { Screenings Onderzoek }\end{array}$ \\
\hline$\rho_{c}$ & concordance correlation coefficient \\
\hline $\operatorname{Perc}_{15}$ & $\begin{array}{l}\text { 15th percentile of the attenuation } \\
\text { curve }\end{array}$ \\
\hline Pi & internal perimeter \\
\hline RB1 & right upper lobe \\
\hline $\mathrm{RVC}_{-860}$ to -950 & $\begin{array}{l}\text { change in relative lung volume with } \\
\text { attenuation values between }-860 \text { and } \\
-950 \mathrm{HU}\end{array}$ \\
\hline $\mathrm{D}$ & standard deviation \\
\hline NA & wall area \\
\hline
\end{tabular}

\section{Introduction}

The use of computed tomography (CT) increases rapidly, resulting in a marked increase in radiation exposure for the population [1]. Therefore, radiation dose saving has received much attention and has been pursued by introducing low-dose protocols using the conventional filtered backprojection (FBP) algorithm for image reconstruction. However, the constraint on radiation dose increases image noise [2]. Recent advances in computational power allowed the introduction of iterative reconstruction (IR) algorithms for image reconstruction. Data suggest that IR allows radiation dose reduction by $50 \%$ or more compared to standard dose acquisition, while maintaining image quality [3-6]. Such a dose reduction would be a major step forward, especially in case of repeated evaluations and follow-up as regularly applied in chest imaging. However, the influence of IR on quantitative CT measurements, e.g. measurement of lung density and airway dimensions used in the evaluation of chronic obstructive pulmonary disease (COPD), is not yet known. Therefore, the aim of this study was to determine the influence of IR on quantitative CT measurements of pulmonary emphysema, air trapping and airway dimensions, compared to the standard FBP algorithm.

\section{Materials and methods}

\section{Subjects}

This study was performed in subjects participating in the population-based Dutch and Belgian randomized lung cancer screening trial (NELSON trial). Inclusion criteria of the trial and study population characteristics have previously been described in detail [7]. Briefly, participants were at baseline current and former smokers (who quit no more than 10 years ago) between the age of 50 and 75 years, who smoked more than 15 cigarettes per day during more than 25 years or more than 10 cigarettes per day during more than 30 years. In the present study we included 83 consecutive subjects who received a paired inspiratory and expiratory CT between June 2011 and August 2011 for lung cancer screening purposes. All CTs were reconstructed using both standard FBP and IR. We excluded a total of eight subjects owing to $\mathrm{CT}$ protocol violation $(n=1)$, postoperative changes after lobectomy of the right upper lobe $(n=1)$, and failure of the automatic lung segmentation $(n=6)$ (see Sect. "Quantitative analysis of emphysema and air trapping"). The final study population thus comprised 75 subjects.

CT data acquisition and image reconstruction

Chest CT was performed using one of two available CT systems: 44 subjects were examined using 64-slice CT (Brilliance 64; Philips Healthcare, Best, the Netherlands) with a smooth reconstruction filter (C-filter, Philips); 31 subjects were examined using 256-slice CT (Brilliance iCT; Philips Healthcare, Best, the Netherlands) using either a smooth B-filter $(n=24)$ or C-filter $(n=7)$. Slices of $1 \mathrm{~mm}$ thickness with $0.7-\mathrm{mm}$ increment were reconstructed. Dose settings were adjusted to patients body weight: $120 \mathrm{kVp}$ at $30 \mathrm{mAs}$ for inspiratory $\mathrm{CT}$ and $80 \mathrm{kVp}$ at $30 \mathrm{mAs}$ for expiratory $\mathrm{CT}$ in subjects weighing less than $80 \mathrm{~kg}$, and $140 \mathrm{kVp}$ at $30 \mathrm{mAs}$ for inspiratory $\mathrm{CT}$ and $120 \mathrm{kVp}$ at $20 \mathrm{mAs}$ for expiratory CT in subjects weighing $80 \mathrm{~kg}$ or more.

Raw CT data of the study subjects were reconstructed using both standard FBP and hybrid IR (iDose; Philips Healthcare, Best, the Netherlands). iDose is a recently introduced reconstruction algorithm using two denoising components $[3,6,8]$, which provides image noise reduction without changing the image characteristics. Technically, iDose applies an iterative maximum likelihood denoising algorithm, based on Poisson statistics, on the raw projection data. Subsequently, the reconstructed images are iteratively adjusted in order to decrease uncorrelated noise. The level of noise reduction is adjustable by selecting one of seven levels (with level 1 having the least noise reduction, and level 7 having the most noise reduction). iDose level 6 was used in the present study, resulting in a theoretical noise reduction of $45 \%$ compared to FBP [8].

Quantitative analysis of emphysema and air trapping

Specialized software automatically segmented the lungs from the chest wall, mediastinum, diaphragm and airways 
[9] in the inspiratory and expiratory CT images of both reconstruction algorithms. Additionally, all lung segmentation results were visually checked and those with major errors excluded, as previously described [10]. Attenuation of each voxel within the segmented lung volume was assessed, and several commonly used CT emphysema and CT air trapping measures were calculated from the attenuation distribution histogram. CT emphysema was defined as the percentage of voxels below $-950 \mathrm{HU}$ in inspiratory CT (IN-950) [11] and as the Hounsfield unit (HU) value at the 15 th percentile of the attenuation curve $\left(\operatorname{Perc}_{15}\right)$ [12]. CT air trapping was defined as the percentage of voxels below -856 HU in expiratory CT $\left(\mathrm{EXP}_{-856}\right)$ [13], the change in relative lung volume with attenuation values between -860 and $-950 \mathrm{HU}\left(\mathrm{RVC}_{-860}\right.$ to -950$)$ [14], and as the expiratory to inspiratory ratio of the mean lung density (E/I-ratio ${ }_{M L D}$ ) [15].

Quantitative analysis of airway dimensions

We used the apical segmental bronchus of the right upper lobe (RB1) to assess airway dimensions [16]. Airway dimensions of RB1 were measured using validated custom software based on the full-width-at-half maximum method (EmphylxJ; University of British Columbia, Vancouver, BC, Canada) $[16,17]$. In short, the RB1 was visually identified on the inspiratory CT by a trained observer, who manually placed a seed point in the lumen centre. The software then calculates the $\mathrm{x}$-ray attenuation along rays placed from the lumen centre outwards in all directions. The airway boundaries are assumed halfway to the maximum on the lumen side, and halfway to the minimum on the parenchymal side $[17,18]$. Using these airway wall boundaries we calculated absolute values of lumen area (LA), wall area (WA) and internal perimeter $(\mathrm{Pi})$ of the RB1 for each subject. Additionally, wall area was expressed as percentage of total airway area: $100 \% \times \mathrm{WA} /(\mathrm{WA}+\mathrm{LA})=\mathrm{WA} \%$. The airway measurements were performed similarly and at the exact same location in both inspiratory CT series.

\section{Data analysis}

Quantitative CT measures of emphysema, air trapping and airway dimensions for the conventional FBP and the IR algorithm were compared within each subject. The agreement of the quantitative CT measures using the two algorithms was assessed by the concordance correlation coefficient $\left(p_{\mathrm{c}}\right)$, which takes into account both the correlation and the distance to the line of identity [19]. A $p_{\mathrm{c}}$ value less than 0.90 was considered to represent poor agreement, whereas higher $p_{\mathrm{c}}$ values represent moderate $\left(0.90 \leq p_{\mathrm{c}} \leq 0.95\right)$, substantial $\left(0.95<p_{\mathrm{c}} \leq 0.99\right)$ or almost perfect $\left(p_{\mathrm{c}}>0.99\right)$ agreement, based on the descriptive scale for continuous variables [20]. The non-parametric Wilcoxon signed rank test was used in all variables to test for statistical differences within the subjects.

Statistical analyses were performed using SPSS software v15.0 (SPSS Inc, Chicago, Illinois, USA) and MedCalc v11.3.8.0, Mariakerke Belgium. A $P$ value below 0.05 was considered statistically significant. Values given are medians with interquartile range, unless indicated otherwise.

\section{Results}

Subjects in our study population were on average $64.0 \pm 5.7$ (SD) years of age, and male $(n=37,49 \%)$ and female subjects $(n=38,51 \%)$ were equally represented.

\section{Comparison of quantitative CT measures}

Comparison of quantitative CT measures using FBP and IR showed significant differences for all CT emphysema measurements. Also most CT air trapping measures differed significantly between FBP and IR, except for the E/Iratio $_{\text {MLD }}$ as a measure of CT air trapping. Finally, airway measurements showed no significant differences between the algorithms. Table 1 lists the quantitative results per reconstruction algorithm. The absolute differences in CT emphysema were $3.04 \%$ (interquartile range 1.86-4.62) for $\mathrm{IN}_{-950}$ and $11 \mathrm{HU}$ (interquartile range 10-13) for Perc 15 . The absolute differences in CT air trapping were $8.0 \%$ (interquartile range 6.1-11.2) for $\mathrm{EXP}_{-856}$ and $7.6 \%$ (interquartile range $4.2-10.2$ ) for $\mathrm{RVC}_{-860}$ to -950 . Except for $\mathrm{E} / \mathrm{I}-$ ratio $_{\mathrm{MLD}}$, all $\mathrm{CT}$ measures of emphysema and air trapping showed poor agreement between standard FBP and IR. CT measures of the apical segmental bronchus of the right upper lobe showed on average substantial agreement. The results of quantitative CT assessment using both reconstruction algorithms are presented in Figs. 1 and 2, further illustrating the systematic differences. Figure 3 shows an example of quantitative assessment of CT emphysema using either the conventional FBP and the IR algorithm.

\section{Discussion}

This study found that noise-reducing IR significantly alters most of the quantitative measures of CT emphysema and CT air trapping generally used in respiratory research. However,

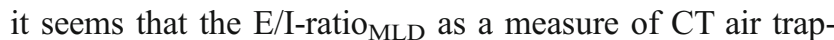
ping and quantitative measurements of a relatively large airway lumen and wall remain unchanged between the two reconstruction algorithms. These findings may be important given that CT quantification of the lungs is increasingly used, while dose reduction and IR methods are introduced at the same time. 
Table 1 Differences in quantitative CT measurements of emphysema, air trapping and airway dimensions using filtered back-projection (FBP) and iDose reconstruction algorithms

\begin{tabular}{|c|c|c|c|c|}
\hline & FBP & iDose & $p_{\mathrm{c}}$ value & $P$ value \\
\hline \multicolumn{5}{|l|}{ CT emphysema } \\
\hline $\mathrm{IN}_{-950}(\%)$ & $3.81(2.17-7.46)$ & $0.57(0.25-2.26)$ & 0.486 & $<0.001$ \\
\hline $\operatorname{Perc}_{15}(\mathrm{HU})$ & $-918(-907$ to -931$)$ & $-906(-896$ to -920$)$ & 0.866 & $<0.001$ \\
\hline \multicolumn{5}{|l|}{ CT air trapping } \\
\hline $\mathrm{EXP}_{-856}(\%)$ & $24.3(17.0-32.6)$ & $14.4(7.4-22.3)$ & 0.777 & $<0.001$ \\
\hline $\mathrm{RVC}_{-860}$ to $-950(\%)$ & $-35.3(-43.1$ to -26.3$)$ & $-42.6(-54.7$ to -30.6$)$ & 0.873 & $<0.001$ \\
\hline E/I-ratio ${ }_{M L D}(\%)$ & 87.9 (83.9-90.9) & $88.1(84.3-90.8)$ & 0.998 & NS \\
\hline \multicolumn{5}{|l|}{ Airway measurements } \\
\hline Lumen area $\left(\mathrm{mm}^{2}\right)$ & $10.3(7.6-14.6)$ & $10.2(7.3-14.5)$ & 0.991 & NS \\
\hline Wall area $\left(\mathrm{mm}^{2}\right)$ & $34.9(29.4-41.7)$ & $34.4(28.8-44.3)$ & 0.960 & NS \\
\hline WA \% (\%) & $77.0(73.1-81.4)$ & $77.6(73.4-80.7)$ & 0.935 & NS \\
\hline $\mathrm{Pi}(\mathrm{mm})$ & $11.8(10.2-13.8)$ & $11.7(10.0-13.7)$ & 0.990 & NS \\
\hline
\end{tabular}

Values given are median with interquartile range

$H U$ Hounsfield units; $I N_{-950} \mathrm{CT}$ emphysema as percentage of voxels below $-950 \mathrm{HU} ;$ Perc $_{15} \mathrm{CT}$ emphysema as 15 th percentile of attenuation distribution curve; $E X P_{-856} \mathrm{CT}$ air trapping as percentage of voxels below $-856 \mathrm{HU} ; R V C_{-860}$ to $-950 \mathrm{CT}$ air trapping as relative change in lung volume with attenuation between -860 and $-950 \mathrm{HU} ;$ E/I-ratio $_{M L D}$ expiratory to inspiratory ratio of mean lung density; WA\% wall area percentage as $100 \% \times \mathrm{WA} /(\mathrm{WA}+\mathrm{LA}) ; P i$ internal perimeter of the airway; $p_{c}$ value concordance correlation coefficient, a correlation $<0.90$ represents poor agreement (see Figs. 1 and 2 for a visual representation); NS not significant

Previous studies have shown that quantitative CT emphysema measures are influenced by several technical factors such as slice thickness $[21,22]$ and type of CT equipment used [23]. However, research into the factors which may
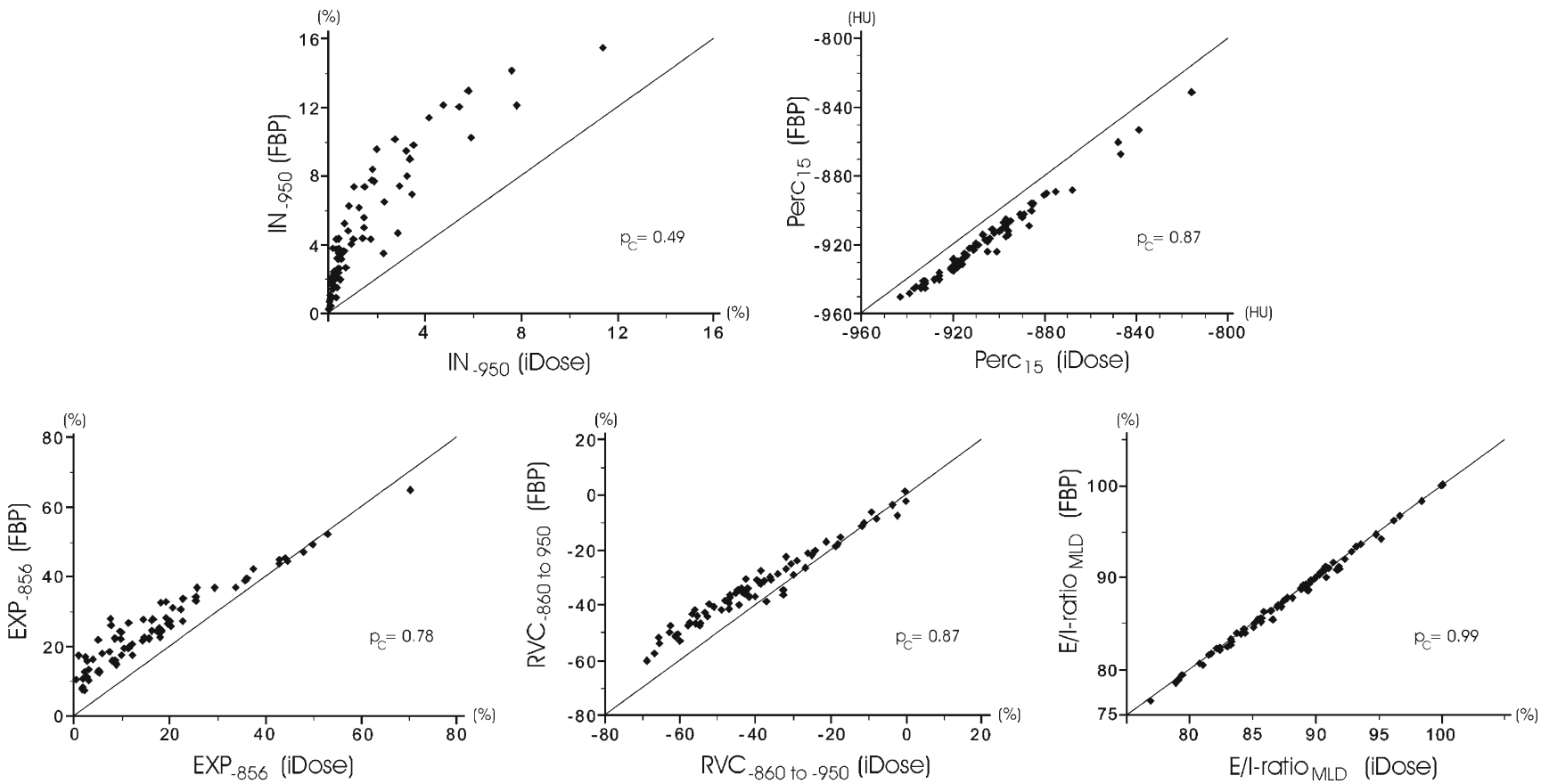

Fig. 1 Scatter plots of quantitative CT measures of emphysema and $\mathrm{CT}$ air trapping using conventional filtered back-projection (FBP) and hybrid iterative reconstruction (iDose). Structural differences between iDose and FBP are shown for CT emphysema as percentage of voxels below $-950 \mathrm{HU}\left(\mathrm{IN}_{-950}\right)$ and as 15 th percentile of attenuation distribution curve $\left(\mathrm{Perc}_{15}\right)$ (upper row) and $\mathrm{CT}$ air trapping as percentage of voxels below $-856 \mathrm{HU}\left(\mathrm{EXP}_{-856}\right)$ and as relative change in lung volume with attenuation between -860 and $-950 \mathrm{HU}$ ( $\mathrm{RVC}_{-860}$ to -950) (lower left and middle). The only quantitative CT measure with a concordance correlation coefficient $\left(p_{\mathrm{c}}\right)$ of at least 0.90 , and thus insensitivity to the iterative reconstruction, is the expiratory to inspiratory ratio of mean lung density (E/I-ratio ${ }_{\mathrm{MLD}}$, lower right) 

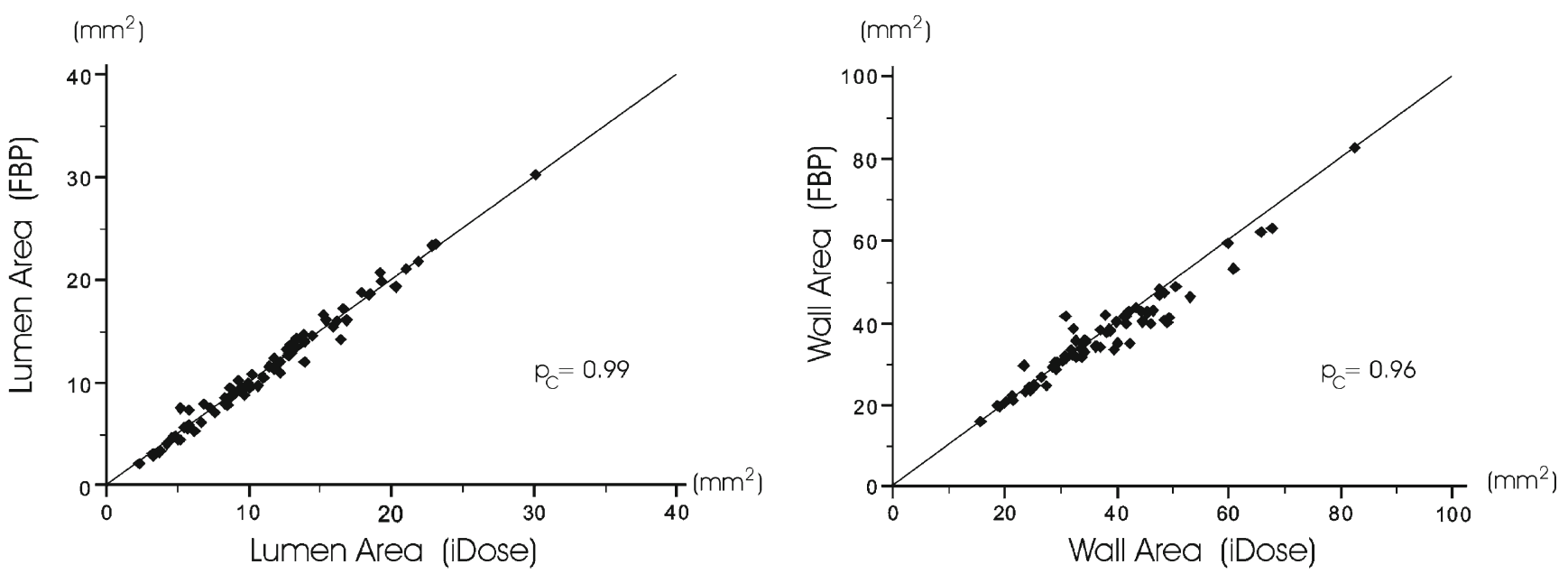

(\%)

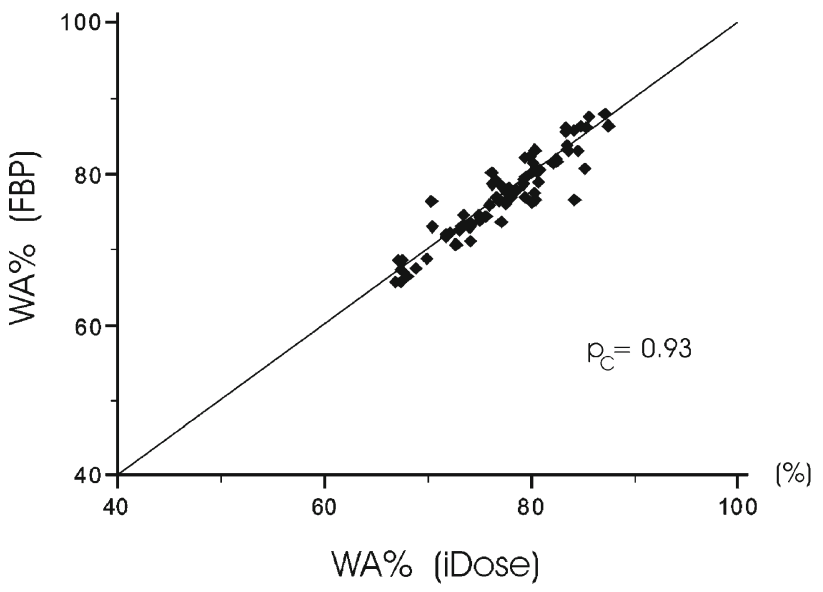

Fig. 2 Scatter plots of quantitative CT measures of the apical segmental bronchus of the right upper lobe using conventional filtered backprojection (FBP) and hybrid iterative reconstruction (iDose). No structural differences between iDose and FBP were found for lumen area

influence CT air trapping assessment and airway measurements has been limited [18]. Our study offers insight into the influence of IR in several widely used quantitative CT measures of emphysema, air trapping and airway dimensions. Given the significant differences that we have shown between the two reconstruction methods for several measures, our findings underline that comparison of quantitative CT results of lung densitometry should always be performed with careful attention to the protocols used for $\mathrm{CT}$ data acquisition and image reconstruction/analysis [18, 24].

Regarding the application of CT air trapping, our findings suggest that $\mathrm{E} / \mathrm{I}-$ ratio $_{\mathrm{MLD}}$ is the preferred method given its insensitivity to differences in the evaluated reconstruction algorithms. This insensitivity may be due to the fact that denoising in IR affects the extremes of the attenuation distribution histogram. As a consequence, threshold methods (e.g. $\mathrm{EXP}_{-856}$ or $\mathrm{RVC}_{-860}$ to -950 for $\mathrm{CT}$ air trapping) are substantially altered whereas the mean lung density is hardly

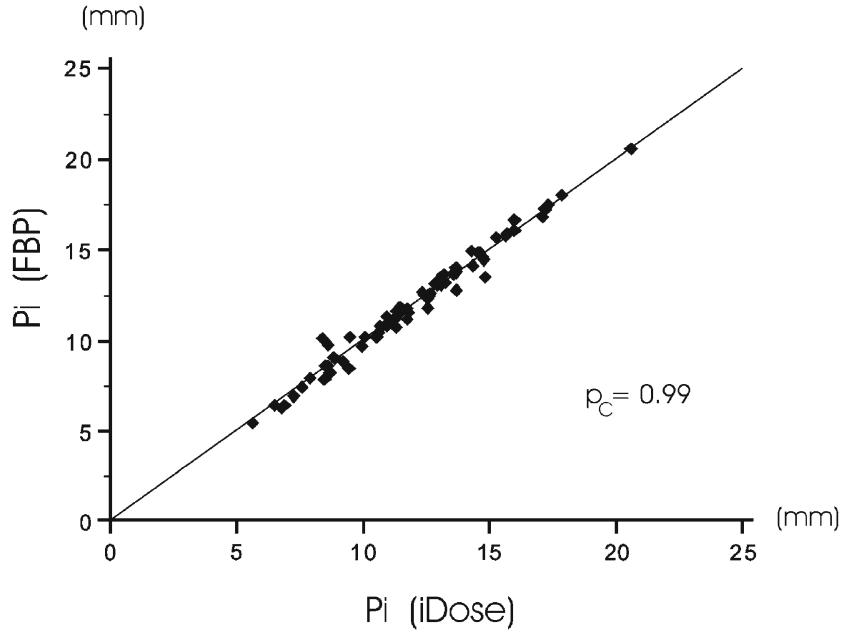

(upper left), wall area (upper right), wall area percentage (WA\%, lower left) and internal perimeter $(\mathrm{Pi}$, lower right), because all concordance correlation coefficients $\left(p_{\mathrm{c}}\right)$ were at least 0.90

affected and this in combination with the use of an inspiratory to expiratory ratio apparently makes this measure independent of a denoising algorithm. If this insensitivity also applies to other protocol differences, such as $\mathrm{kVp}$ and $\mathrm{mAs}$, this might imply that $\mathrm{E} / \mathrm{I}-$ ratio $_{\mathrm{MLD}}$ is preferable over other CT air trapping measures.

Further, our findings suggest that the denoising process in IR does not affect the delineation of segmental airway structures, given that the differences in airway measurements for the right apical segmental bronchus between both reconstruction methods were not significantly different from zero.

Our study has potential limitations. Firstly, it is important to note the lack of a pathological reference standard, which would be needed to judge which method is close to a 'pathological truth', although we would like to emphasize that this study specifically aimed to investigate and describe the differences that occur when IR is applied instead of 


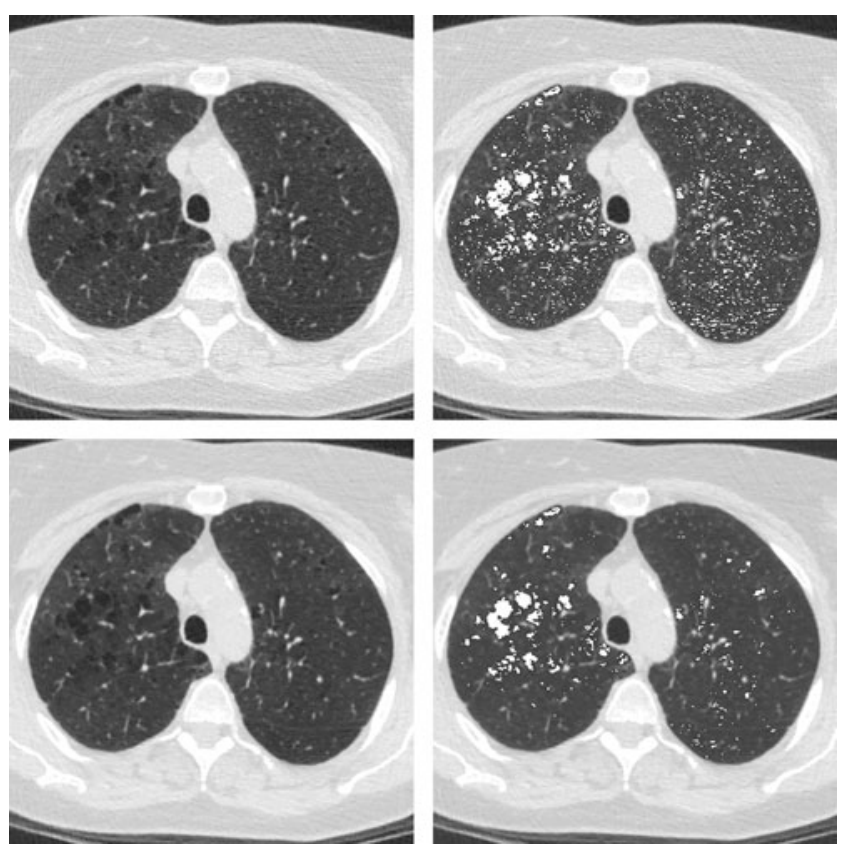

Fig. 3 Quantitative assessment of CT emphysema using the conventional filtered back-projection (FBP) and iterative reconstruction algorithm (iDose). Axial CT images in inspiration. The lungs are automatically segmented from the chest wall, airways and mediastinum using dedicated software. Attenuation of each voxel in the segmented lung volume is calculated and CT emphysema is defined as voxels with an attenuation below $-950 \mathrm{HU}$; voxels within this range are coloured white (right images). Note the denoising effect and the difference in CT emphysema between the FBP (upper images) and iDose (lower images)

conventional FBP. Secondly, it is noted that our results might differ between altering IR denoising levels and other IR algorithms and CT manufacturers, given that the results were obtained from testing the IR algorithm of a single vendor at one denoising level. Future research might focus on ways to correct for structural differences in quantitative measures when IR algorithms are applied. Thirdly, we focussed on a commonly used segmental airway and on the basis of our findings it cannot be concluded that measurements on smaller airways are unaffected by IR.

In conclusion, our study shows that the evaluated IR algorithm significantly alters quantitative CT measures in the assessment of all emphysema and most commonly used air trapping measures, compared to FBP. However, both the E/I-ratio ${ }_{M L D}$ as a measure of CT air trapping and the quantitative measurements in a segmental airway are unaffected by this reconstruction method. Quantitative CT lung densitometry should always be performed with careful attention to the CT protocol, especially in an era of increased use of quantitative $\mathrm{CT}$ where dose reduction and iterative reconstruction are introduced.

Acknowledgments We thank K. Thijn, A. Manders, J. Mounmi and R. Valkenburg for technical assistance.
The NELSON trial was sponsored by the Netherlands Organisation for Health Research and Development (ZonMw); Dutch Cancer Society Koningin Wilhelmina Fonds (KWF); Stichting Centraal Fonds Reserves van Voormalig Vrijwillige Ziekenfondsverzekeringen (RvvZ); Siemens Germany; Rotterdam Oncologic Thoracic Steering committee (ROTS); G.Ph.Verhagen Trust, Flemish League Against Cancer, Foundation Against Cancer and Erasmus Trust Fund.

The funders had no role in study design, data collection and analysis, decision to publish, or preparation of the manuscript.

Open Access This article is distributed under the terms of the Creative Commons Attribution License which permits any use, distribution, and reproduction in any medium, provided the original author(s) and the source are credited.

\section{References}

1. Brenner DJ, Hall EJ (2007) Computed tomography-an increasing source of radiation exposure. N Engl J Med 357:2277-2284

2. Yuan R, Mayo JR, Hogg JC et al (2007) The effects of radiation dose and CT manufacturer on measurements of lung densitometry. Chest 132:617-623

3. Funama Y, Taguchi K, Utsunomiya D et al (2011) Combination of a low-tube-voltage technique with hybrid iterative reconstruction (iDose) algorithm at coronary computed tomographic angiography. J Comput Assist Tomogr 35:480-485

4. Winklehner A, Karlo C, Puippe G et al (2011) Raw data-based iterative reconstruction in body CTA: evaluation of radiation dose saving potential. Eur Radiol 21:2521-2526

5. Sagara Y, Hara AK, Pavlicek W, Silva AC, Paden RG, Wu Q (2010) Abdominal CT: comparison of low-dose CT with adaptive statistical iterative reconstruction and routine-dose CT with filtered back projection in 53 patients. AJR Am J Roentgenol 195:713-719

6. Habets J, Symersky P, de Mol BA, Mali WP, Leiner T, Budde RP (2011) A novel iterative reconstruction algorithm allows reduced dose multidetector-row CT imaging of mechanical prosthetic heart valves. Int J Cardiovasc Imaging. doi:10.1007/s10554-011-9954-7

7. van Iersel CA, de Koning HJ, Draisma G et al (2007) Risk-based selection from the general population in a screening trial: selection criteria, recruitment and power for the Dutch-Belgian randomised lung cancer multi-slice CT screening trial (NELSON). Int J Cancer 120:868-874

8. Noel PB, Fingerle AA, Renger B, Munzel D, Rummeny EJ, Dobritz M (2011) Initial performance characterization of a clinical noise-suppressing reconstruction algorithm for MDCT. AJR Am J Roentgenol 197:1404-1409

9. van Rikxoort EM, de Hoop B, Viergever MA, Prokop M, van Ginneken B (2009) Automatic lung segmentation from thoracic computed tomography scans using a hybrid approach with error detection. Med Phys 36:2934-2947

10. Mets OM, Murphy K, Zanen P et al (2012) The relationship between lung function impairment and quantitative computed tomography in chronic obstructive pulmonary disease. Eur Radiol $22: 120-128$

11. Gevenois PA, de Maertelaer V, De Vuyst P, Zanen J, Yernault JC (1995) Comparison of computed density and macroscopic morphometry in pulmonary emphysema. Am J Respir Crit Care Med 152:653-657

12. Newell JD Jr, Hogg JC, Snider GL (2004) Report of a workshop: quantitative computed tomography scanning in longitudinal studies of emphysema. Eur Respir J 23:769-775

13. Regan EA, Hokanson JE, Murphy JR et al (2010) Genetic epidemiology of COPD (COPDGene) study design. COPD 7:32-43 
14. Matsuoka S, Kurihara Y, Yagihashi K, Hoshino M, Watanabe N, Nakajima Y (2008) Quantitative assessment of air trapping in chronic obstructive pulmonary disease using inspiratory and expiratory volumetric MDCT. AJR Am J Roentgenol 190:762-769

15. O'Donnell RA, Peebles C, Ward JA et al (2004) Relationship between peripheral airway dysfunction, airway obstruction, and neutrophilic inflammation in COPD. Thorax 59:837-842

16. Nakano Y, Muro S, Sakai H et al (2000) Computed tomographic measurements of airway dimensions and emphysema in smokers. Correlation with lung function. Am J Respir Crit Care Med 162:1102-1108

17. Nakano Y, Whittall KP, Kalloger SE, Coxson H, Pare PD, English JC (2002) Development and validation of human airway analysis algorithm using multidetector row CT. Proc SPIE 4683:460-469

18. Mets OM, de Jong PA, van Ginneken B, Gietema HA, Lammers JW (2012) Quantitative computed tomography in COPD: possibilities and limitations. Lung 190:133-145
19. Lin LI (1989) A concordance correlation coefficient to evaluate reproducibility. Biometrics 45:255-268

20. McBride GB (2005) A proposal for strength-of-agreement criteria for Lin's concordance correlation coefficient. NIWA Client Report: HAM2005-062. http://www.medcalc.org/download/pdf/ McBride2005.pdf. Accessed 14 May 2012

21. Madani A, Zanen J, De Maertelaer V, Gevenois PA (2006) Pulmonary emphysema: objective quantification at multi-detector row CT - comparison with macroscopic and microscopic morphometry. Radiology 238:1036-1043

22. Gierada DS, Bierhals AJ, Choong CK et al (2010) Effects of CT section thickness and reconstruction kernel on emphysema quantification relationship to the magnitude of the CT emphysema index. Acad Radiol 17:146-156

23. Bakker ME, Stolk J, Putter H et al (2005) Variability in densitometric assessment of pulmonary emphysema with computed tomography. Invest Radiol 40:777-783

24. Coxson HO, Rogers RM (2005) Quantitative computed tomography of chronic obstructive pulmonary disease. Acad Radiol 12:1457-1463 